\title{
Characterization of Carbamazepine-Loaded Solid Lipid Nanoparticles Prepared by Rapid Expansion of Supercritical Solution
}

\author{
Zahra Akbari ${ }^{1}$, Massoud Amanlou ${ }^{2}$, Javad Karimi-Sabet ${ }^{3}$, Abolfazl Golestani ${ }^{4}$ \\ and Mojtaba Shariaty Niasar ${ }^{5}$ \\ ${ }^{1}$ School of Chemical Engineering, Collage of Engineering, University of Tehran, ${ }^{2}$ Department of Medicinal Chemistry, Faculty of \\ Pharmacy, Tehran University of Medical Sciences, ${ }^{3}$ Jaber Ebne Hayyan National Research Laboratory, NSTRI, ${ }^{4}$ Department of \\ Biochemistry, Tehran University of Medical Sciences, ${ }^{5}$ School of Chemical Engineering, Collage of Engineering, University of \\ Tehran, Tehran, Iran
}

*For correspondence: Email: zakbary@ut.ac.ir; Tel: +989126161892

Received: 19 June 2014

Revised accepted: 24 October 2014

\begin{abstract}
Purpose: To prepare carbamazepine-loaded solid lipid nanoparticles (CBZ-SLN) for prolonged release of $C B Z$ in oral drug delivery.

Methods: CBZ-loaded SLNS were prepared by rapid expansion of supercritical solution (RESS). SLNs were formulated using stearic acid as the lipid component. Initially, lipid and drug were separately micronized by RESS process separately. This was followed by co-precipitation of the components with the two solutes using RESS to produce drug-loaded SLNs. The formulations were characterized by infrared spectroscopy (IR), scanning electron microscopy (SEM) and ultraviolet spectrophotometry (UV). Results: Application of RESS to pure stearic acid yielded spherical particles in the range 40 to $200 \mathrm{~nm}$ which was about 600 times smaller than the unprocessed powder based on SEM observation. Similarly, RESS processing of carbamazepine produced nanoparticles with rod shape. FT-IR analysis showed that no chemical structure change occurred during RESS processing of both components. Coprecipitation of drug and lipid using RESS produced SLNs with drug loading capacity of $2.2 \%$. RESS yielded ultrafine spherical particles $(100 \mathrm{~nm})$ of CBZ-SLNs.

Conclusion: The successful preparation and characterization of carbamazepine-loaded solid lipid nanoparticles by RESS as well as their characterization has been achieved in this study.
\end{abstract}

Keywords: Rapid expansion of supercritical fluid, Stearic acid, Solid lipid nanoparticles, Carbamazepine, Co-precipitation

Tropical Journal of Pharmaceutical Research is indexed by Science Citation Index (SciSearch), Scopus, International Pharmaceutical Abstract, Chemical Abstracts, Embase, Index Copernicus, EBSCO, African Index Medicus, JournalSeek, Journal Citation Reports/Science Edition, Directory of Open Access Journals (DOAJ), African Journal Online, Bioline International, Open-J-Gate and Pharmacy Abstracts

\section{INTRODUCTION}

Drug-loaded solid lipid nanoparticles (SLNs) have attracted increasing attention in recent years as an efficient drug delivery system. SLN has many advantageous such as controlled drug release, non-biotoxicity of the carrier, increased bioavailability of drug and lower overall cost [1-4]. Current methods to produce drug-loaded SLNs include high-pressure homogenization (hot or cold), microemulsion technique, emulsification/evaporation and solvent emulsification/ diffusion [5-7].

Rapid Expansion of Supercritical Solution (RESS) is a new technique for SLN production and has several advantages such as (i) avoidance of the use of solvents; (ii) particles are 
obtained as a dry powder, instead of suspensions, and (iii) mild pressure and temperature conditions are applied [8]. In RESS process, a solution in the supercritical state is expanded rapidly through a capillary nozzle. Due to rapid changes in density and therefore solvent power, the solution becomes highly supersaturated and consequently particles of high purity with small sizes are formed [9]. Much work has been done on producing particles of pure compounds by RESS [10], but only a few studies have been undertaken on the simultaneous co-precipitation of two components. Also to the best of our knowledge, no studies have been published on the formation of drug-loaded SLNs with RESS technique.

In this study, at first, stearic acid (SA) as lipid and carbamazepine (CBZ) as drug were micronized by RESS process separately. Based on results of exploratory experiments, the feasibility of preparing carbamazepine loaded solid lipid nanoparticle using CO-RESS process was evaluated. The physicochemical properties of obtained CBZ and SA nanoparticles and composite powder of CBZ loaded SLNs, such as surface properties, morphology, loading and chemical structure were characterized by Scanning electron microscopy (SEM), spectrophotometry and fourier transform infrared spectroscopy (FTIR) respectively.

\section{EXPERIMENTAL}

\section{Materials}

Stearic acid (SA, purity: $99.9 \%$, MW: 284.48, Merck ChemicalCo.) was used as lipid for SLN production. Carbamazepine supplied from Arasto Company (CBZ, purity: $99.9 \%$, MW: 236.269). Carbon dioxide with the purity of $99.99 \%$ was purchased from Farafan Gas Company which was used as extracting agent. Potassium Bromide was supplied from Merck. It should be stated that all the chemicals were used as received without any further purification.

\section{Apparatus}

The RESS apparatus is shown schematically in Figure 1. The system consists of two sections: extraction and precipitation. A high accurate temperature-controlled water bath with an uncertainty of $\pm 0.1 \mathrm{~K}$ was used (Type WPE 45 , Memmert Germany) to keep the temperature of the equilibrium cells constant. The capacity of the equilibrium cells placed in the water bath, vessels 4 and 5 in the schematic diagram, was 200 and $300 \mathrm{~cm}^{3}$, respectively. The body of the equilibrium cells, valves, and tubes were all made of stainless steel and supplied from Swagelok Company (USA). Two vessels were used in series in order to ensure that equilibrium conditions were achieved. The cells were filled with stearic acid at various temperature and pressure. The pressure of the cells was measured with a digital pressure transducer with an uncertainty of $\pm 0.1 \mathrm{MPa}$ (Type KM11, Ashcroft Instruments, Germany). Purified gaseous $\mathrm{CO} 2$ was liquefied and subcooled in a chiller and thereafter compressed to the desired pressure with a reciprocating pump type SFT-10 (Supercritical Fluid Technologies Inc, USA). Sinter metal filter was used on both ends of vessels 4 and 5 to avoid flooding of the undissolved material imposed by $\mathrm{CO}_{2}$ flow.

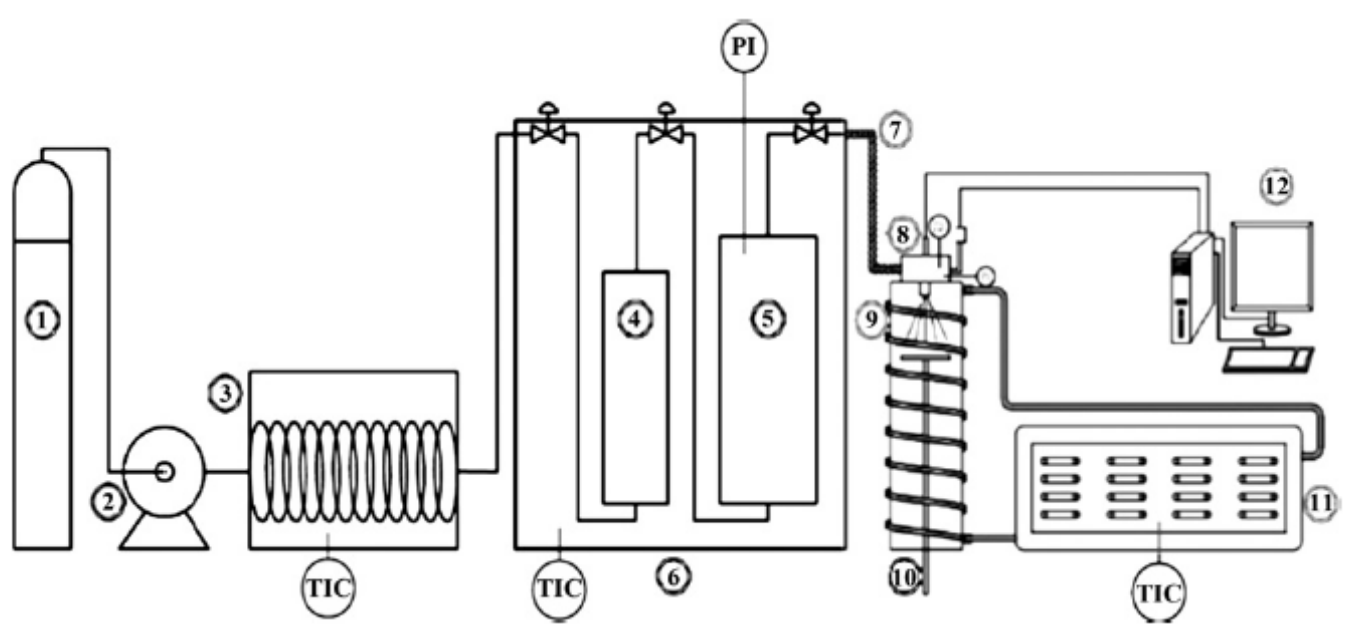

Figure 1: The experimental set-up for RESS process. (1) $\mathrm{CO}_{2}$ tank, (2) pump, (3) heat exchanger, $(4,5)$ equilibrium vessels, (6) constant temperature water bath, (7) preheating tube, (8) expansion device, (9) 
precipitation unit, (10) collection sample holder, (11) refrigerated water bath circulator, (12) data acquisition system (TI: temperature indicator; TIC: temperature indicator controller; $\mathrm{PI}$ : pressure indicator) Preheating coils and the extraction vessel were immersed at constant temperature water bath. The precipitation unit consists of a cell, an expansion device and a collection device. Equipment of material is made of aluminum. The main part of the expansion device is a nozzle (SITEC Co, Switzerland). During rapid expansion and in order to avoid freezing and plugging within the nozzle that is heated by a cartridge heater, which is controlled by a PID controller (Type TPR- 2N, Han Young NUX Co, Republic of Korea). The temperature of the gas flowing through the nozzle was measured by a thermocouple (J-type) and a digital display (Type TPR-2N, Han Young NUX Co, Republic of Korea). The thermocouple was placed precisely into the solution stream approximately $7 \mathrm{~mm}$ above the entrance region of the nozzle. A highly accurate temperature-controlled refrigerated water bath was used to keep the temperature of the precipitation unit constant (Type WCL-P8, Daihan Scientific Co, Republic of Korea). The collection device, by which the precipitated particles are collected, is placed inside the precipitation cell. The top part of the collection device, namely the collection table, is made of Teflon. For collection of the particles, the glass slides are located on this table. The spray distance (the distance between the tip of the nozzle and the surface of the glass slide) can be changed via adjustable bolt and nut mechanism. In previous studies, this apparatus has been used for nanoparticles production [11]

\section{Characterization}

The morphology and size of the precipitated particles were examined by SEM (KYKY, EM3200 , China). For analysis, the particles were attached to the carbon tape that is on the top of SEM aluminum stubs and were then coated with gold using a sputter coater. The SEM figures which were taken from the collected glass slide were introduced to the image processing software. All SEM analyses were done at accelerating voltage of 24 and $26 \mathrm{kV}$. Chemical analyses of samples were performed by FTIR spectrophotometer (Bruker- Tenasor 27, Germany). About 1-2 mg of sample was mixed with dry potassium bromide and the samples were examined at transmission mode over wave number range of 4,000 to $400 \mathrm{~cm}^{-1}$. The amount of $\mathrm{CBZ}$ in drug loaded solid lipid nanoparticles was determined using UV spectrophotometer (WTW 6600, Germany). Accurate weight of SLN composite powder was dissolved in the $10 \mathrm{ml}$ of methanol. A CBZ calibration curve at concentrations varying from 5 to $80 \mathrm{mg} / \mathrm{mL}$ was used for analysis.

\section{Statistical analysis}

The data were expressed as mean \pm standard deviation (SD). Statistical analysis for determination of particle size distribution was performed using image analysis software (Digimizer). All the tests were run in triplicate. The level of significance used was $p<0.05$.

\section{Particle formation}

Preliminary experiments were carried out in order to precipitate pure lipid, pure drug and mixture of them in the mass ratio of $1: 1$ and 1:3 (SA: CBZ). In each experiment, $2 \mathrm{~g}$ (additional material to ensure the attaining of equilibrium conditions) of sample was charged into each equilibrium vessel. It is worth pointing out that glass granular beads were introduced into the equilibrium vessel in association with the chemicals to increase the contact surface area. Prior to running the experiments, entrapped air was purged out from the vessels and piping by $\mathrm{CO}_{2}$ injection. In all experiments, nozzle diameter is $45 \mu$ and the spray distance is $5 \mathrm{~cm}$ and time of gathering precipitated particles on glass slides is $20 \mathrm{~min}$.

\section{RESULTS}

\section{Stearic acid nanoparticles}

First, exploratory experiment was carried out in order to investigate the formation of SA nanoparticles. This experiment was performed at pre-expansion temperature $50{ }^{\circ} \mathrm{C}$, extraction pressure 100 bar and extraction temperature 35 ${ }^{\circ} \mathrm{C}$. Figure 2 shows a typical SEM and FTIR images of unprocessed SA and micronized SA produced by RESS process. Unprocessed SA particles have a mean size of $100 \pm 36.7$ um and are nearly spherical in shape illustrated in Figure 2(a). After RESS process, the generated SA nanoparticles exhibited a smaller mean diameter size of $142 \pm 30 \mathrm{~nm}$ as illustrated in Figure 2(b). The results show that SA particles were produced with narrow particle size distribution. Also, the SEM image shows a modification of the morphology of the precipitated particles.

The principal FT-IR peak of plain SA and SA nanoparticles obtained at $1701 \mathrm{~cm}^{-1}$ is due to the absorption of $\mathrm{C}=\mathrm{O}$ stretching group (Figure 2(c)). The spectrum shows the other characteristic 

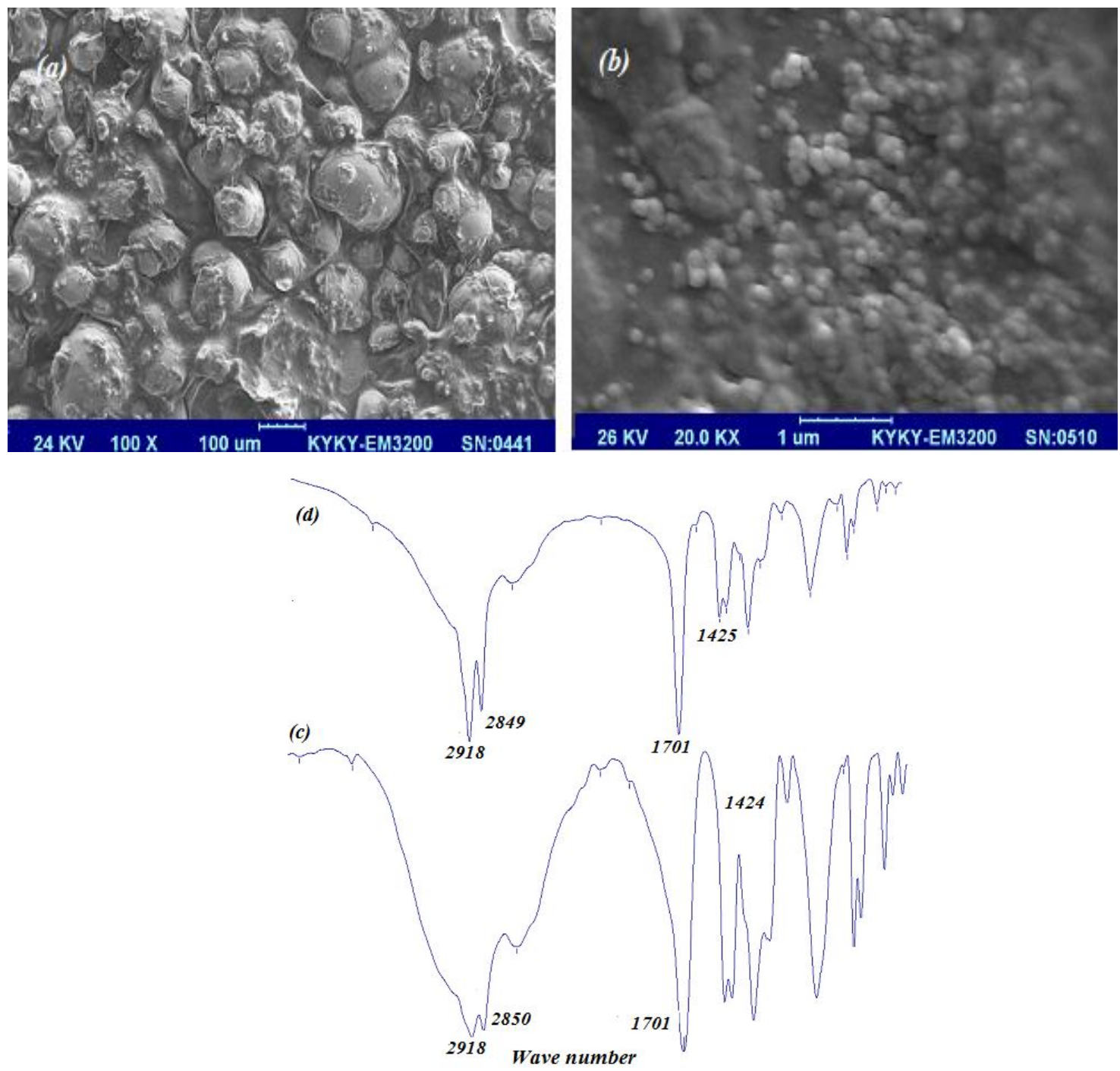

Figure 2: (a) SEM image of unprocessed SA particles (b) TSEM image of processed SA particles after RESS at extraction pressure of $100 \mathrm{bar}$, extraction temperature of $35{ }^{\circ} \mathrm{C}$ and pre-expansion temperature of $50 \circ \mathrm{C}$; FTIR spectra of (c) unprocessed SA particles, and (d) processed SA particles prepared by RESS at extraction pressure of 100 bar, extraction temperature of $35^{\circ} \mathrm{C}$ and pre-expansion temperature of $50{ }^{\circ} \mathrm{C}$

peak of SA such as hydrogen-bonded $\mathrm{O}-\mathrm{H}$ stretching at $2850 \mathrm{~cm}^{-1}$ which have obscured other peaks in this region. The other principal peaks obtained are $2918 \mathrm{~cm}^{-1}$ (C-H stretching) and $1424 \mathrm{~cm}^{-1}-1500 \mathrm{~cm}^{-1}$ (C-H bend).

As illustrated in Figure 2(d), in the spectrum of micronized SA, principal peaks were obtained at $1702 \mathrm{~cm}^{-1} \quad(\mathrm{C}=\mathrm{O}$ stretching), $2849 \quad(\mathrm{O}-\mathrm{H}$ stretching) and $2919 \mathrm{~cm}^{-1}$ (aliphatic C-H stretching). Since pre-expansion temperature is greater than the melting temperature of stearic acid, liquid droplets were formed and quickly solidified within the expansion unit, leading to the formation of larger. Therefore, pre-expansion temperature should be less than the melting temperature of micronized SA [12].

\section{Carbamazepine nanoparticles}

The RESS experiment for CBZ nanoparticle preparation was carried out at pre-expansion temperature $100{ }^{\circ} \mathrm{C}$, extraction pressure 200 bar and extraction temperature $60{ }^{\circ} \mathrm{C}$. Typical SEM images of unprocessed and RESS processed drug are depicted in Figure 3(a) and 3(b). SEM images show that average particles size of raw CBZ and CBZ nanoparticles were $30.00 \pm 8.29$ um and $400.00 \pm 36.25 \mathrm{~nm}$, respectively. The results show that $C B Z$ particles were produced with narrow particle size distribution. Figures present the prismatic morphology of raw material composed of large particles of CBZ and needleshape of CBZ nanoparticles. FT-IR spectrum of raw and micronized $C B Z$ are illustrated in Figure $3(c)$ and (d). 

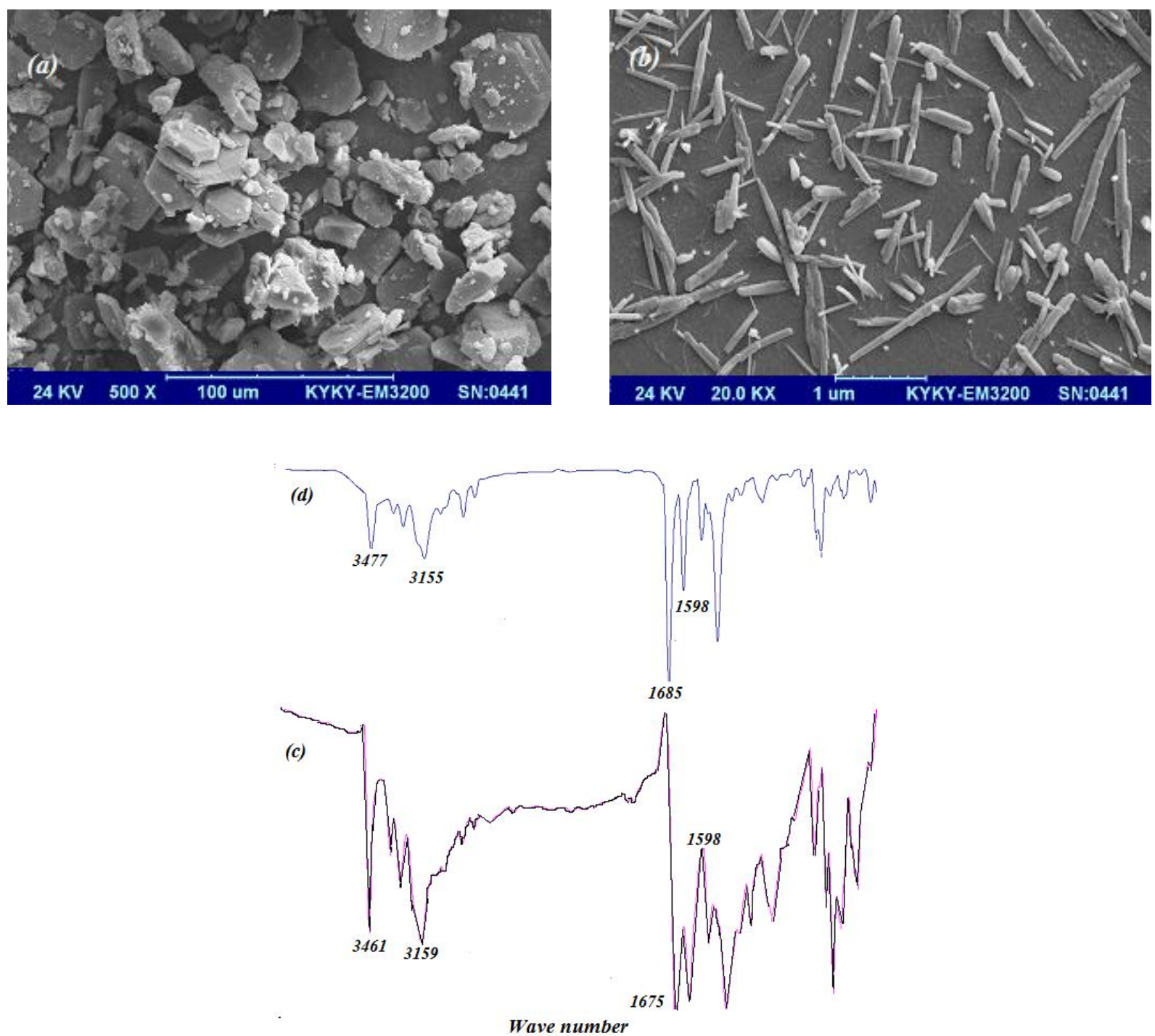

Figure 3: SEM image of (a) unprocessed CBZ particles; (b) processed CBZ particles prepared by RESS at extraction pressure of 200 bar, extraction temperature of $60 \circ \mathrm{C}$ and pre-expansion temperature of $100 \circ \mathrm{C}$; FTIR spectra of (c) unprocessed CBZ particles, and (d) processed CBZ particles prepared by the RESS extraction pressure of 200 bar, extraction temperature of $60{ }^{\circ} \mathrm{C}$ and pre-expansion temperature of $100^{\circ} \mathrm{C}$

In the spectra of unprocessed $\mathrm{CBZ}$ and micronized $\mathrm{CBZ}$, principal peaks were obtained at $1675 \mathrm{~cm}^{-1}$ and $1685 \mathrm{~cm}^{-1}$ for $\mathrm{C}=\mathrm{O}$ stretching, $1598 \mathrm{~cm}^{-1}$ and $1598 \mathrm{~cm}^{-1}$ for $\mathrm{C}=\mathrm{C}$ stretching, $3159 \mathrm{~cm}^{-1}$ and $3155 \mathrm{~cm}^{-1}$ for aromatic $\mathrm{C}-\mathrm{H}$ stretching and $3461 \mathrm{~cm}^{-1}$ and $3477 \mathrm{~cm}^{-1}$ for $\mathrm{N}-\mathrm{H}$ stretching. The results also show that small changes in absorption frequency were observed in the spectra of CBZ nanoparticles compared to unprocessed CBZ.

\section{Formation of nanocomposite}

Based on the results obtained for the pure substances, SA and CBZ, composite particles were produced by the simultaneous coprecipitation of two solutes in RESS process.
Physical mixture of the two solutes with the mass ratio of 1:1 and 3:1 (drug: lipid) were applied. The physical mixtures were prepared by weighing out and thoroughly mixing the required amount of $\mathrm{CBZ}$ and SA. Mixture was then mixed with glass granular beads and packed into columns. CORESS experiments carried out at an extraction temperature of $40{ }^{\circ} \mathrm{C}$, extraction pressure of 100 bar, and pre-expansion temperature of $50^{\circ} \mathrm{C}$.

Figure 4 shows respectively the FT-IR spectra of drug loaded SA nanoparticles samples with different initial mass ratio of drug and lipid (1:1, 3:1). Also, in Table 1, interpretation of the FT-IR spectra and their functional peaks are listed. The presence of characteristic peaks of SA and CBZ 
in drug-loaded SLN reveals that co-precipitation of drug and lipid was occurred.

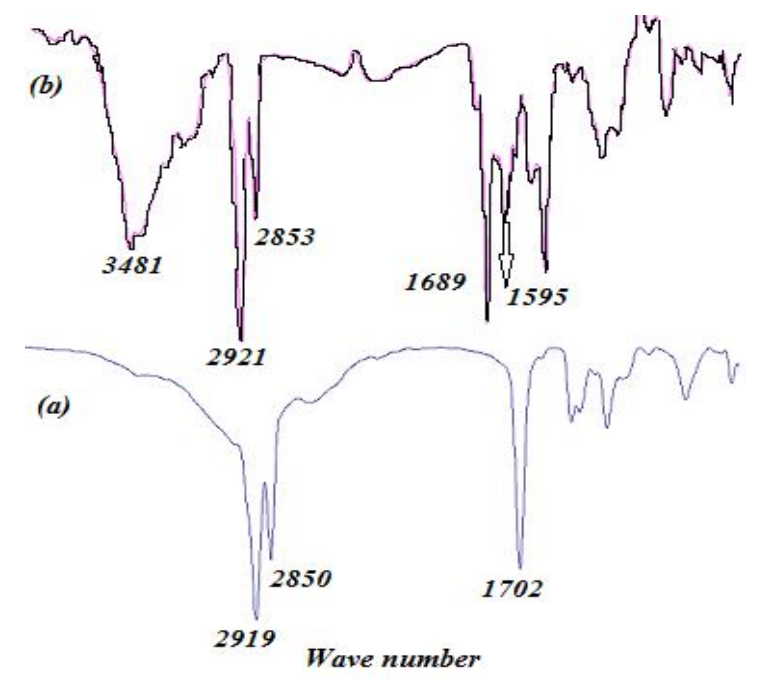

Figure 4: FT-IR spectra of (a) CBZ-loaded SA nanoparticles (1:1), and (b) CBZ-loaded SA nanoparticles (3:1)

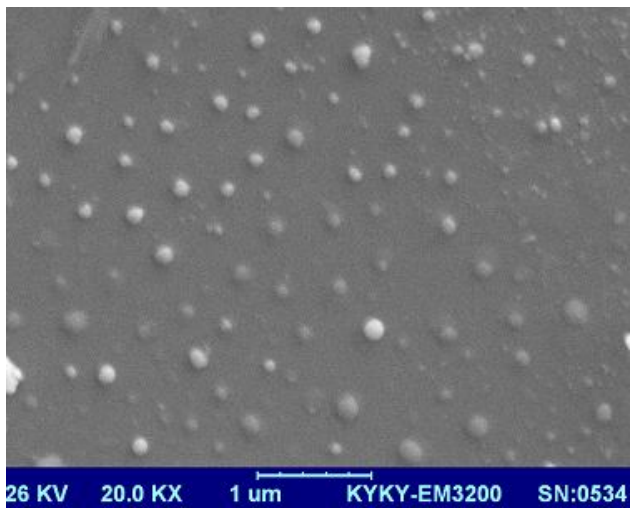

Fig 5: SEM image of CBZ loaded SA nanoparticles prepared by the RESS at extraction pressure of 100 bar, extraction temperature of $40{ }^{\circ} \mathrm{C}$ and preexpansion temperature of $45^{\circ} \mathrm{C}$

$\mathrm{CBZ}$ and $\mathrm{SA}$, which were subject to $\mathrm{SC}-\mathrm{CO}_{2}$ at the extraction temperature of $40{ }^{\circ} \mathrm{C}$, extraction pressure of 100 bar and pre-expansion temperature of $45^{\circ} \mathrm{C}$, exhibited a modification of the morphology of the precipitated particles illustrated as shown in Figure 5. The mean particle size of drug-loaded stearic acid nanoparticles was $100.00 \pm 10.55 \mathrm{~nm}$. Drug incorporation efficiency (DE) in CBZ-loaded SA nanoparticles is computed as in Eq 1.

$\mathrm{DE}(\%)=(\mathrm{A} / \mathrm{M}) 100$

where $A$ is the amount of drug in the precipitated particles and $M$ is the weight of precipitated particles.

The amount of CBZ in samples was determined by UV spectrophotometer. The wavelength of maximum absorbance of CBZ dissolved in methanol is $285 \mathrm{~nm}$. CBZ calibration curve (correlation coefficient was 0.99) at concentrations varying from 0 to $80 \mathrm{mg} / \mathrm{mL}$ was used for analysis.

\section{DISCUSSION}

The results on SA nanoparticle formation by RESS show that there was a slight change in the particle morphology and a sharp decrease in particle size after processing with RESS but it was less agglomerated. Due to fair solubility of $\mathrm{SA}$ in supercritical $\mathrm{CO}_{2}$, increase in extraction temperature or pressure would lead to greater agglomeration of SA nanoparticles. A comparison of principal FT-IR peaks of unprocessed and processed SA indicate that there was no change in chemical structure of stearic acid under high pressure in RESS process.

The SEM image of CBZ nanoparticles indicate that the crystal structure of CBZ has changed completely during the RESS process. CBZ has four polymorphs. Crystal shape of polymorph I has not been identified. Polymorph II and III are respectively triclinic and monoclinic crystal with prismatic morphology and polymorph IV presents needle-shape morphology [13]. Therefore, SEM images of raw CBZ and CBZ nanoparticles show that polymorphism occurred during RESS process due to change of particles morphology.

Table 1: Interpretation of FT-IR spectra of raw drug and lipid, CBZ-loaded SLN, and physical mixture of CBZ and lipid

\begin{tabular}{lcccc}
\hline Functional group & Drug & Lipid & CBZ-SLN (1:1) & CBZ-SLN (3:1) \\
\hline N-H stretching & 3461 & - & - & 3481 \\
C=O stretching & 1598 & 1701 & 1702 & 1689 \\
aromatic C-H stretching & 3159 & - & - & - \\
C=C stretching & 1667 & - & - & - \\
C-N & 1383 & - & - & 1397 \\
O-H Stretching & - & 2849 & 2850 & 2853 \\
aliphatic C-H stretching & - & 2918 & 2919 & 2921 \\
\hline
\end{tabular}


Furthermore, there is a sharp decrease in CBZ particle size after processing with RESS. The results from FTIR studies of CBZ nanoparticles are interesting. The small shift in absorption frequency was observed in the FTIR spectra of CBZ nanoparticles. From the comparative study of nano-materials and bulk materials, the difference in the frequency of vibrational modes is attributed to dipolar interactions, interfacial effects, surface amorphousness and surface free energy [14]. The chemical structure of CBZ was not changed during RESS process because no new peaks were observed in the FTIR spectra. Due to the low solubility of CBZ in SCF- $-\mathrm{CO}_{2}$, CBZ were subjected to high extraction pressure and temperature in $\mathrm{CBZ}$ nanoparticle production.

The absence of characteristic peaks of $C B Z$ in CBZ-loaded SLN (1:1) suggest that coprecipitation of drug and lipid did not occur in the extraction unit only, as only SA dissolved in SCF$\mathrm{CO}_{2}$. The results show that CBZ-loaded SLNs (3:1) showed peaks resulting from simple superposition of their separated components in the infrared spectra. The presence of characteristic peaks of SA and CBZ reveals that the specific functional groups of lipid material in the nanoparticles surface have almost the same chemical characteristics of the pure lipid. The study suggests that molecular interactions that could alter the chemical structure of the CBZ did not occur. It is evident that the particles have a distinct spherical shape with smooth surface and precipitated particles have a mean size of $100,000 \pm 10.55 \mathrm{~nm}$ as reflected by SEM observations. The results show that drug loading in CBZ loaded SLN (3:1) is $2.2 \%$. As mentioned above, this is because of low solubility of CBZ in $\mathrm{SC}-\mathrm{CO}_{2}$. Hence, optimum drug incorporation efficiency can be tunable with initial mass ratio of the physical mixture of drug and lipid.

\section{CONCLUSION}

This study demonstrates that rapid expansion of supercritical solution can be used to produce submicron composite particles of CBZ loaded SLN. Based on these results, co-precipitation of drug and lipid with different initial mass ratio were carried out by RESS process. The results also show that spherical particles of CBZ-loaded SLNs can be produced with a drug loading capacity of $2.2 \%$.

\section{REFERENCES}

1. Pardeshi C, Rajput P, Belgamwar V, Tekade A, Patil G, Chaudhary $K$, Sonje A. Solid lipid based nanocarriers: An overview. Acta Pharma 2012; 62: 433-472.

2. Mehnert W, Mader K. Solid lipid nanoparticles Production, characterization and applications. Adv Drug Del Rev 2001; 47: 165-196.

3. Reddy RN, Shariff A, Solid lipid Nanoparticles: An Advanced Drug Delivery System, Int J Pharm Sci Res 2013; 4(1): 161-171.

4. Nair R, Kumar AC, Priya VK, Yadav CM, Raju PY. Formulation and evaluation of chitosan solid lipid nanoparticles of carbamazepine. Lipids in Health and Disease 2012; 11: 72-81.

5. Hippalgaonkar K, Majumdar S, Kansara V. Injectable lipid emulsions advancements, opportunities and challenges. AAPS Pharm Sci Tech 2010; 11: 15261540.

6. Wang $Y$, Zhu L, Dong Z, Xie S, Chen X, Lu M, Wang X, Li X, Zhoua WZ. Preparation and stability study of norfloxacin-loaded solid lipid nanoparticle. Suspensions Colloids and Surfaces $B$ : Biointerfaces2012; 98:105- 111.

7. Ekambaram P, Abdul Hasan Sathali A, Priyanka K. Solid Lipid Nanoparticle: A Review. Sci Rev Chem Comm 2012; 2: 80-102.

8. Pawar Bhima B, Gavale Chandrakant S, Akrte Anup M, Baviskar Dheeraj T. Solid lipid nanoparticles; the beneficial carrier for the delivery of lipid soluble drugs. Int J pharma Res Dev 2011; 2: 200 - 209.

9. Reverchon E, Adami R. Nanomaterials and supercritical fluids. J Supercritical Fluids 2006; 37:1-22. J Supercritical Fluids 2009; 47: 546-555.

10. Cocero MJ, Martín A, Varona FS. Encapsulation and coprecipitation processes with supercritical fluids: Fundamentals and applications. $J$ Supercritical Fluids 2009; 47: 546-555.

11. Keshavarz A, Karimi-sabet J, Fattahi A, Golzary AA, Rafiee-Tehrani M, Dorkoosh FA. Preparation and characterization of raloxifene nanoparticles using Rapid Expansion of Supercritical Solution (RESS). J Supercritical Fluids 2012; 63: 169-179.

12. Akbari Z, Amanlou M, Karimi-Sabet J, Golestaniand A, Shariaty Niassar M. Preparation and Characterization of Stearic Acid Nanoparticles through Rapid Expansion of Supercritical Solution. Int J Pharma Sci Res 2014; 5(5): 1-11.

13. Grzesiak A L, Lang M, Kim K, Matzger A.J. Comparison of the Four Anhydrous Polymorphs of Carbamazepine and the Crystal Structure of Form I. J Pharma Sci 2003; 92(11): 2260-2271.

14. Available from: URL: http://dspace.nitrkl.ac.in/dspace/ bitstream/2080/1632/1/Characterisation.pdf 